\title{
Subthalamic nucleus deep brain stimulation for Parkinson's disease: evidence for effectiveness and limitations from 12 years' experience
}

\author{
Movement Disorder Group; Anne YY Chan, Jonas HM Yeung, Vincent CT Mok, Vincent HL Ip, \\ Adrian Wong, SH Kuo, Danny TM Chan, XL Zhu, Edith Wong, Claire KY Lau, Rosanna KM Wong, \\ Venus Tang, Christine Lau, WS Poon *
}

\section{A B S T R A C T}

Objective: To present the result and experience of subthalamic nucleus deep brain stimulation for Parkinson's disease.

Design: Case series.

Setting: Prince of Wales Hospital, Hong Kong.

Patients: A cohort of patients with Parkinson's disease received subthalamic nucleus deep brain stimulation from September 1998 to January 2010. Patient assessment data before and after the operation were collected prospectively.

Results: Forty-one patients ( 21 male and 20 female) with Parkinson's disease underwent bilateral subthalamic nucleus deep brain stimulation and were followed up for a median interval of 12 months. For the whole group, the mean improvements of Unified Parkinson's Disease Rating Scale (UPDRS) parts II and III were $32.5 \%$ and $31.5 \%$, respectively $(\mathrm{P}<0.001)$. Throughout the years, a multidisciplinary team was gradually built. The deep brain stimulation protocol evolved and was substantiated by updated patient selection criteria and outcome assessment, integrated imaging and neurophysiological targeting, refinement of surgical technique as well as the accumulation of experience in deep brain stimulation programming. Most of the structural improvement occurred before mid-2005. Patients receiving the operation before June 2005 (19 cases) and after (22 cases) were compared; the improvements in UPDRS part III were $13.2 \%$ and $55.2 \%$, respectively $(\mathrm{P}<0.001)$. There were three operative complications (one lead migration, one cerebral haematoma, and one infection) in the group operated on before 2005. There was no operative mortality.

This article was published on 24 Oct 2014 at www.hkmj.org.

Conclusions: The functional state of Parkinson's disease patients with motor disabilities refractory to best medical treatment improved significantly after subthalamic nucleus deep brain stimulation. A dedicated multidisciplinary team building, refined protocol for patient selection and assessment, improvement of targeting methods, meticulous surgical technique, and experience in programming are the key factors contributing to the improved outcome.

\section{Hong Kong Med J 2014;20:474-80}

DOI: 10.12809/hkmj144242

Movement Disorder Group, Prince of Wales Hospital, The Chinese University of Hong Kong, Shatin, Hong Kong

${ }^{1} \mathrm{AYY}$ Chan, MB, ChB, MRCP

${ }^{2}$ JHM Yeung, MB, ChB, MRCP

${ }^{1}$ VCT Mok, MD

${ }^{1}$ VHL Ip, MB, ChB, MRCP

${ }^{1} \mathrm{~A}$ Wong, PhD

${ }^{3}$ SH Kuo, MD

${ }^{4}$ DTM Chan, FRCS (Edin), FHKAM (Surgery)

${ }^{4}$ XL Zhu, FRCS (Edin), FHKAM (Surgery)

${ }^{4} \mathrm{E}$ Wong, BSC

${ }^{4}$ CKY Lau, MSc

${ }^{5}$ RKM Wong, MSc

${ }^{4,6} \mathrm{~V}$ Tang, PhD

${ }^{1} \mathrm{C}$ Lau, MSc

${ }^{4}$ WS Poon *, FHKAM (Medicine)

1 Division of Neurology, Department of Medicine and Therapeutics, Prince of Wales Hospital, The Chinese University of Hong Kong, Hong Kong Division of Neurology, Department of Medicine, Alice Ho Miu Ling Nethersole Hospital, Hong Kong

Neurological Institutes of New York, Columbia University, United States Division of Neurosurgery, Department of Surgery, Prince of Wales Hospital, The Chinese University of Hong Kong, Hong Kong

${ }^{5}$ Department of Occupational Therapy, Prince of Wales Hospital, The Chinese University of Hong Kong, Hong Kong

${ }^{6}$ Department of Clinical Psychology, Prince of Wales Hospital, Hospital Authority, Hong Kong

* Corresponding author: wpoon@cuhk.edu.hk

New knowledge added by this study

- The current study provides data on subthalamic nucleus deep brain stimulation (DBS) for Parkinson's disease from a tertiary hospital in Hong Kong and the development of this treatment method over the past 12 years.

Implications for clinical practice or policy

- There was significant improvement in the outcome between the early and later groups of the series. Possible factors contributing to the improvement are dedicated multidisciplinary team building, refinement of the DBS protocol for patient assessment and selection, improvement of targeting methods, meticulous surgical technique, and accumulation of experience in DBS programming. 


\section{Introduction}

Parkinson's disease (PD) is the second most common neurodegenerative disorder that initially affects the dopaminergic system and eventually involves other neurotransmitter systems, with an unrelenting course. It is well known that motor fluctuations like wearing-off and peak-dose dyskinesia are common motor complications few years after patients are started on medical treatment. When these complications become very disabling despite maximal adjustment of pharmacological agents, deep brain stimulation (DBS) has been shown to be effective and safe with lasting benefits for at least up to 10 years..$^{1-5}$ Deep brain stimulation is a neurosurgical intervention that entails inserting microelectrodes with imaging and neurophysiological guidance at targeted regions, such as the subthalamic nucleus (STN) or globus pallidus interna (GPi) in order to achieve alleviation of most of the motor complications. We studied DBS performed in a cohort of PD patients in a single Hong Kong centre over a period of 12 years. Patients' outcomes improved significantly throughout all these years. Here, we review our results of STN DBS for PD and summarise our experience, with particular emphasis on evaluating the effectiveness and safety of this novel treatment.

\section{Methods}

\section{Patients}

Patients who fulfilled the Queen Square Brain Bank Criteria for diagnosis of PD and experienced significant disability due to motor fluctuations despite maximal medical therapy were admitted to the Prince of Wales Hospital and jointly evaluated for the suitability for DBS by a Movement Disorder Group composed of neurologists, neurosurgeons, and a nurse specialist. After June 2005, this multidisciplinary group was further developed to include a radiologist, clinical psychologist, occupational therapist, physiotherapist, and speech therapist. The assessment comprised a predefined protocol, which was continuously updated over the years. This assessment protocol included clinical assessment, levodopa challenge test, video recording, neuroimaging, and neurocognitive and psychiatric evaluations. Patients who received STN DBS from September 1998 (the first patient who received STN DBS in our centre) to January 2010 were included in the present study. Assessment data were obtained before and after the operation (medication-off state and stimulator-on) and included the Unified Parkinson's Disease Rating Scale (UPDRS), modified Hoehn-and-Yahr stage (both at PD off-state), levodopa equivalent dose, body weight, and patients' diaries at preoperation and at least 2 months postoperation.

\section{丘腦下核深部腦電刺激治療柏金遜病的效果與 局限性：12年經驗分享}

運動障礙組；陳然欣、楊漢明、莫仲棠、葉慶龍、黃沛霖、 郭昇翰、陳達明、朱獻倫、黃秀蘭、劉嘉怡、黃潔汶、 鄧如卿、劉妍姬、潘偉生

目的：報導丘腦下核（STN）深部腦電刺激（DBS）治療柏金遜病的 效果及經驗。

設計：病例研究。

安排：香港威爾斯親王醫院。

患者：1998年9月至2010年1月期間所有接受STN DBS的柏金遜病 例, 並前瞻性採集其術前和術後的評估資料。

結果：共41例（分別21男20女）柏金遜患者接受STN DBS治療。隨 訪中位數為 12 個月。整個組別的統一柏金遜病評估量表（UPDRS）

II和III的改善率分別為 $32.5 \%$ 和 $31.5 \%(\mathrm{P}<0.001)$ 。研究期間逐漸 組成一個多學科團隊, 並制定了一套DBS的方案, 包括病人選擇、 綜合成像和神經生理定靶, 以及手術前後的評估標準。通過經驗累積 不斷改良手術和程控方法。上述大部份結構性改善均在2005年中以 前發生。比較2005年6月前（19例）及後（22例）兩組病人的治療結 果, UPDRS III的改善率分別為 $13.2 \%$ 和 $55.2 \% （ \mathrm{P}<0.001 ） 。 2005$ 年 前分別有電極移位、臚內血腫和感染各一例手術併發症發生。全組無 手術相關的死亡病例。

結論：當最好的內科治療未能對柏金遜患者的活動障礙殘疾奏效 時, STN DBS能顯著改善患者的活動功能。一個專職的多學科團隊、 優化的患者選擇和評估方案、精確的靶點定位、精細的手術和程控經 驗累積是提高療效的關鍵。

\section{Surgical information}

All patients had stereotactic guidance for the insertion of electrodes under local anaesthesia. Microelectrode recording (MER) technique was introduced since year 2000. Frame-based stereotaxy was performed in most of the patients except three who underwent frameless stereotaxy (2005) with equally accurate targeting. Before 2003, an old version of Zeppelin magnetic resonance imaging (MRI)-compatible frame was used, which was not compatible with the computer planning system available at that time. Since 2003, a Leksell G frame (Elekta AB, Stockholm, Sweden) was acquired together with a computer planning system (iPlan; Brain LAB, Feldkirchen, Germany) for image targeting and trajectory planning. With these, the image targeting and trajectory were planned based on preoperative MRI. On the day of operation, a computed tomography (CT) scan of brain with the stereotactic frame fixed to the head was performed and fused to the MRI plan in the computer planning system. Such a practice gave us more time for image targeting and shortened the time used for MRI and target planning on the day of operation before entering the operating theatre; this contributed to the accuracy of STN targeting and patient comfort. A dedicated MRI protocol was established since 2008, which ensured the consistency of high-quality MRI 
for targeting. In 2003, microdriver was introduced for MER which provided sub-millimetre advancement of the microelectrode to obtain more details of neuronal discharges and, thus, better quality of MER for neurophysiological targeting. Macrostimulation was performed in all patients for target confirmation. Deep brain stimulation electrode was implanted if satisfactory signals from MER and response from macrostimulation were obtained. For anchoring the DBS lead at the burr-hole site, a reliable device (Navigus; Medtronic, Minneapolis [MN], US) was introduced in 2002. The Pulse Generator (Itrel II or Soletra for unilateral, Kinetra for bilateral; Medtronic, Minneapolis [MN], US) was implanted under general anaesthesia. The DBS was usually switched on within 2 weeks after the operation. A nurse specialist took up DBS programming under the supervision of a neurologist and neurosurgeon in 2004, which facilitated experience accumulation and consistency in DBS programming.

\section{Statistical analyses}

Descriptive data were expressed as mean \pm standard deviation (SD). Wilcoxon test was used to compare scores of modified Hoehn-and-Yahr stage, levodopa equivalent dose, body weight, patients' diaries, as well as scores of individual items of activities of daily living (part II) and motor examination (part III) of UPDRS before and after operation. Tests with a P value of $\leq 0.05$ were considered to be statistically significant. Statistical analyses were performed with the Statistical Package for the Social Sciences (Windows version 15.0; SPSS Inc, Chicago [IL], US).

\section{Results}

Between September 1998 and January 2010, a total of 51 PD patients received DBS. Of them, six received unilateral STN DBS, two received GPi DBS, and two received nucleus ventralis intermedius (VIM) stimulation. Overall, 41 patients received bilateral STN DBS (21 male, 20 female; age at operation: mean 54 , SD 7, range 40-71 years). All were Han Chinese except one who was of Portuguese ethnicity. Mean $( \pm$ SD) duration of PD at operation was $10 \pm 4$ (range, 3-22) years (Table 1 ).

There were three complications, namely, lead migration, infection, and cerebral haematoma; all occurred in 2001. Lead migration occurred in 2001 three months after the operation. The patient complained of increased PD symptoms after a fall on level ground. One DBS lead was found withdrawn from the target. It was related to the insecurity of lead fixation. Revision operation was done for the case with good recovery. Infection occurred in a case 3 weeks after DBS operation in 2001. Apart from abscess formation in the chest wall where the pulse generator was implanted, MRI brain showed contrast
TABLE I. Baseline characteristics of patients undergoing bilateral or sequential subthalamic nucleus deep brain stimulation

\begin{tabular}{lc}
\hline Characteristic & Value \\
\hline Gender & $21(51 \%)$ \\
\hline Male & $20(49 \%)$ \\
\hline Female & \\
\hline Age at operation (years) & $54 \pm 7$ \\
$\quad$ Mean \pm SD & $40-71$ \\
\hline Range & \\
\hline Disease duration at operation (years) & $10 \pm 4$ \\
\hline Mean \pm SD & 9 \\
\hline Median & $3-22$ \\
\hline Range & $16 \pm 15$ \\
\hline Postoperative follow-up duration (months) & 12 \\
\hline Mean \pm SD & $2-77$ \\
\hline Median & $21 \pm 12$ \\
\hline Range & \\
\hline Time elapsed in conducting sequential STN & \\
DBS (months) & \\
\hline Mean \pm SD & \\
\hline Median & \\
\hline Range & \\
\hline
\end{tabular}

Abbreviations: SD = standard deviation; STN DBS = subthalamic nucleus deep brain stimulation

enhancement around the DBS electrodes. All the hardware including the pulse generator and the DBS leads were removed and the patient received a course of antibiotics. He received DBS again in 2006 and enjoyed good effect. Cerebral haematoma also occurred in 2001. The patient became drowsy during the operation. The surgery was stopped and CT brain revealed a subcortical haematoma likely related to venous infarction. Craniotomy for haematoma evacuation was performed. The patient recovered but with residual hemiparesis. There was no mortality.

At postoperative follow-up with a median assessment time of 12 months, both UPDRS parts II and III showed improvements of $32.5 \%$ and $31.5 \%$, respectively $(\mathrm{P}<0.001)$ in the whole study sample (Table 2). In view of the evolvement of the protocol, improvement in targeting methods, refinement of surgical technique as well as accumulation of programming experience over the 12 years, the improvement rates in patients who had DBS from 1999 to May 2005 and from June 2005 to 2010 were analysed separately and compared. Patients in the later group had significantly higher rate of improvement than those in the early group (Table 3 ). The improvement in UPDRS part II and part III for the two groups were $5.1 \%$ vs $55.0 \%(\mathrm{P}=0.005)$ and $13.2 \%$ vs $55.2 \%$, respectively $(\mathrm{P}<0.001)$. The Figure shows the percentage of improvement in UPDRS parts II and 
TABLE 2. Comparisons of UPDRS scores and other parameters before and after bilateral and sequential subthalamic nucleus deep brain stimulation*

\begin{tabular}{|c|c|c|c|}
\hline Measure & Preoperative & Postoperative & $P$ value \\
\hline \multicolumn{4}{|l|}{ UPDRS ADL (part II) } \\
\hline Writing & $2.0 \pm 1.3$ & $1.3 \pm 1.2$ & 0.004 \\
\hline Freezing of gait & $2.3 \pm 1.3$ & $1.1 \pm 1.3$ & $<0.001$ \\
\hline Total & $24.6 \pm 1.0$ & $16.6 \pm 11.8$ & $<0.001$ \\
\hline Improvement & \multicolumn{2}{|c|}{$32.5 \%$} & \\
\hline \multicolumn{4}{|l|}{ UPDRS motor examination (part III) } \\
\hline Tremor & $5.5 \pm 4.6$ & $3.2 \pm 3.7$ & 0.005 \\
\hline Rigidity & $8.0 \pm 5.4$ & $6.0 \pm 4.9$ & 0.006 \\
\hline Akinesia & $16.6 \pm 6.9$ & $8.7 \pm 6.4$ & $<0.001$ \\
\hline Speech & $1.6 \pm 0.8$ & $1.6 \pm 0.8$ & 0.592 \\
\hline Postural stability & $2.0 \pm 1.1$ & $1.8 \pm 1.1$ & 0.046 \\
\hline Gait & $2.3 \pm 1.0$ & $1.7 \pm 1.1$ & 0.003 \\
\hline Total & $44.8 \pm 16.8$ & $30.7 \pm 18.0$ & $<0.001$ \\
\hline Improvement & \multicolumn{2}{|c|}{$31.5 \%$} & \\
\hline UPDRS parts II and III subtotal score & $71.1 \pm 24.8$ & $48.8 \pm 27.8$ & $<0.001$ \\
\hline Improvement & & & \\
\hline \multicolumn{4}{|l|}{ Other parameters } \\
\hline Dose of levodopa-equivalent medication (mg/day) & $882.3 \pm 561.8$ & $708.6 \pm 576.5$ & 0.195 \\
\hline Hoehn-and-Yahr stage (off-stage) & $3.8 \pm 1.1$ & $3.0 \pm 1.4$ & 0.002 \\
\hline Hoehn-and-Yahr stage (on-stage) & $2.0 \pm 0.9$ & $1.8 \pm 1.1$ & 0.1 \\
\hline Body weight & $53.7 \pm 11.5$ & $57.5 \pm 12.2$ & $<0.001$ \\
\hline Diary 'good on' (\% of waking hours) & $37.8 \pm 22.3$ & $55.1 \pm 28.8$ & 0.015 \\
\hline Diary on with dyskinesia (\% of waking hours) & $18.8 \pm 17.7$ & $11.2 \pm 15.7$ & 0.021 \\
\hline Diary off (\% of waking hours) & $43.3 \pm 22.2$ & $32.6 \pm 22.4$ & 0.039 \\
\hline
\end{tabular}

Abbreviations: $A D L=$ activities of daily living; UPDRS = Unified Parkinson's Disease Rating Scale

* Data are shown as mean \pm standard deviation, unless otherwise specified

TABLE 3. Comparison between patients with deep brain stimulation performed before and after June 2005

\begin{tabular}{|c|c|c|c|}
\hline \multirow[t]{2}{*}{ Measure } & \multicolumn{2}{|c|}{ Mean \pm standard deviation } & \multirow[t]{2}{*}{$P$ value } \\
\hline & 1999 to May $2005(n=19)$ & June 2005 to $2010(n=22)$ & \\
\hline Age at operation (years) & $54.1 \pm 9.4$ & $54.2 \pm 5.5$ & 0.961 \\
\hline Disease duration before operation (years) & $10.6 \pm 3.9$ & $8.9 \pm 4.0$ & 0.16 \\
\hline \multicolumn{4}{|l|}{ Preoperative UPDRS } \\
\hline Part II total & $25.0 \pm 12.8$ & $24.8 \pm 5.8$ & 0.944 \\
\hline Part III total & $43.7 \pm 18.1$ & $42.6 \pm 14.8$ & 0.727 \\
\hline \multicolumn{4}{|l|}{ Postoperative UPDRS } \\
\hline Part II total & $22.0 \pm 13.1$ & $11.3 \pm 7.4$ & 0.004 \\
\hline Part III total & $40.4 \pm 18.3$ & $21.0 \pm 11.5$ & $<0.001$ \\
\hline \multicolumn{4}{|l|}{ \% Improvement in UPDRS } \\
\hline Part II total & $5.1 \pm 67.6$ & $55.0 \pm 25.3$ & 0.005 \\
\hline Part III total & $13.2 \pm 31.6$ & $55.2 \pm 18.5$ & $<0.001$ \\
\hline
\end{tabular}

Abbreviation: UPDRS= Unified Parkinson's Disease Rating Scale 
III. There was statistically significant increase in body weight after operation as shown in Table 2. However, there was a non-significant trend with regard to the reduction of levodopa-equivalent daily dose after surgery. A comprehensive neurocognitive evaluation was established after 2008 (Table 4). Three patients completed pre- and post-operative evaluation. Their functioning level in various domains remained unaffected at postoperative evaluation, although a reduction in verbal fluency was noted. No separate statistical analysis was performed due to the limited number of subjects.

As per the patients' own diary reporting, there were statistically significant improvements in "onperiod without dyskinesia" (ie more 'good-on'), "onperiod with dyskinesia", and "off-period" in terms of percentage of waking hours (Table 2).

\section{Discussion}

Parkinson's disease is a neurodegenerative condition typically manifesting in an unrelenting progressive course. Patients often show dramatic response to pharmacological treatments initially, but with time, motor complications like motor fluctuations with wearing-off or peak-dose dyskinesia occur, eventually leading to progressive disability. Deep brain stimulation has evolved as an important and established treatment option for advanced PD. The mechanism of DBS is generally believed to modulate the circuit function by inhibition or excitation through controlled electrical stimulation of different targets along the circuit. Subthalamic nucleus is the most often used target for PD. The benefits of DBS compared with ablative surgery include its nondestructive nature, reversibility, and adjustability. The procedure is mainly indicated for PD patients who are dopamine-responsive but with disabling motor complications such as motor fluctuation, dyskinesia, or intolerable side-effects of anti-PD medications. ${ }^{6}$ To date, there are controversies regarding the use of DBS in the early stage of PD, and most centres will offer this treatment for patients with significant motor complications refractory to maximal drug treatment. Convincing evidence shows that DBS is an effective treatment for PD patients and the benefits may last for at least 10 years.

Our study attempted to investigate the effectiveness and safety of bilateral STN DBS in PD patients who had disabilities refractory to bestavailable medical treatment. The study extends over a period of 10 years, trying to evaluate PD patients who received bilateral STN DBS. The study excluded patients receiving unilateral STN, as well as VIM or GPi DBS, so that the patient group was more homogeneous in the surgical therapeutic sense.

The median timing of postoperative assessment was 12 months (range, 2-77 months), and it demonstrated significant improvement in almost all parameters in UPDRS part II and part III, except for speech. Although our assessments were not performed at the same time intervals for all patients postoperatively, the results are still in concordance with findings in published studies evaluating efficacy of DBS at 6 months and 5 years postoperatively. Speech was also reported to be the only dysfunction that did not improve with STN DBS in published cohorts in general. The exact reason for this finding is yet to be unfolded; a recent paper suggested that speech impairment may be related to unintended activation of dorsal premotor cortex during STN stimulation, ${ }^{7}$ but it echoes with the observation that DBS improves symptoms which respond to levodopa; speech is, however, not one of those.

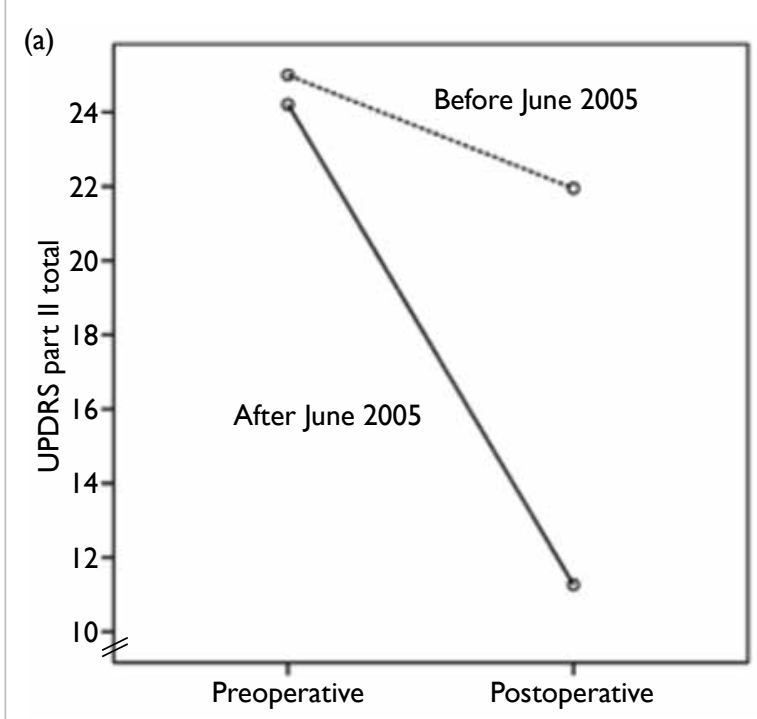

(b)

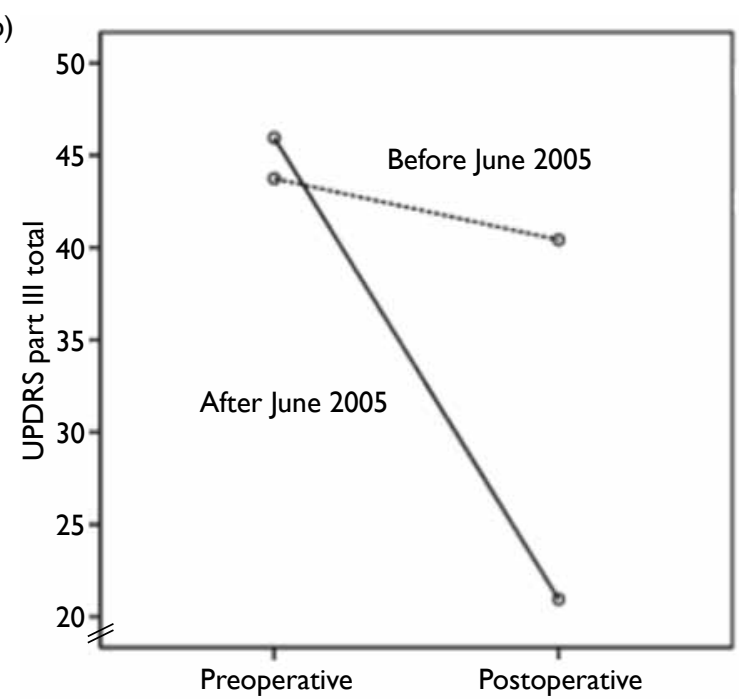

FIG. UPDRS (a) part II and (b) part III in patients with deep brain stimulation performed before or after June 2005 Abbreviation: UPDRS = Unified Parkinson's Disease Rating Scale 
TABLE 4. Protocol for deep brain stimulation for Parkinson's disease (peri-operative management part is not shown)

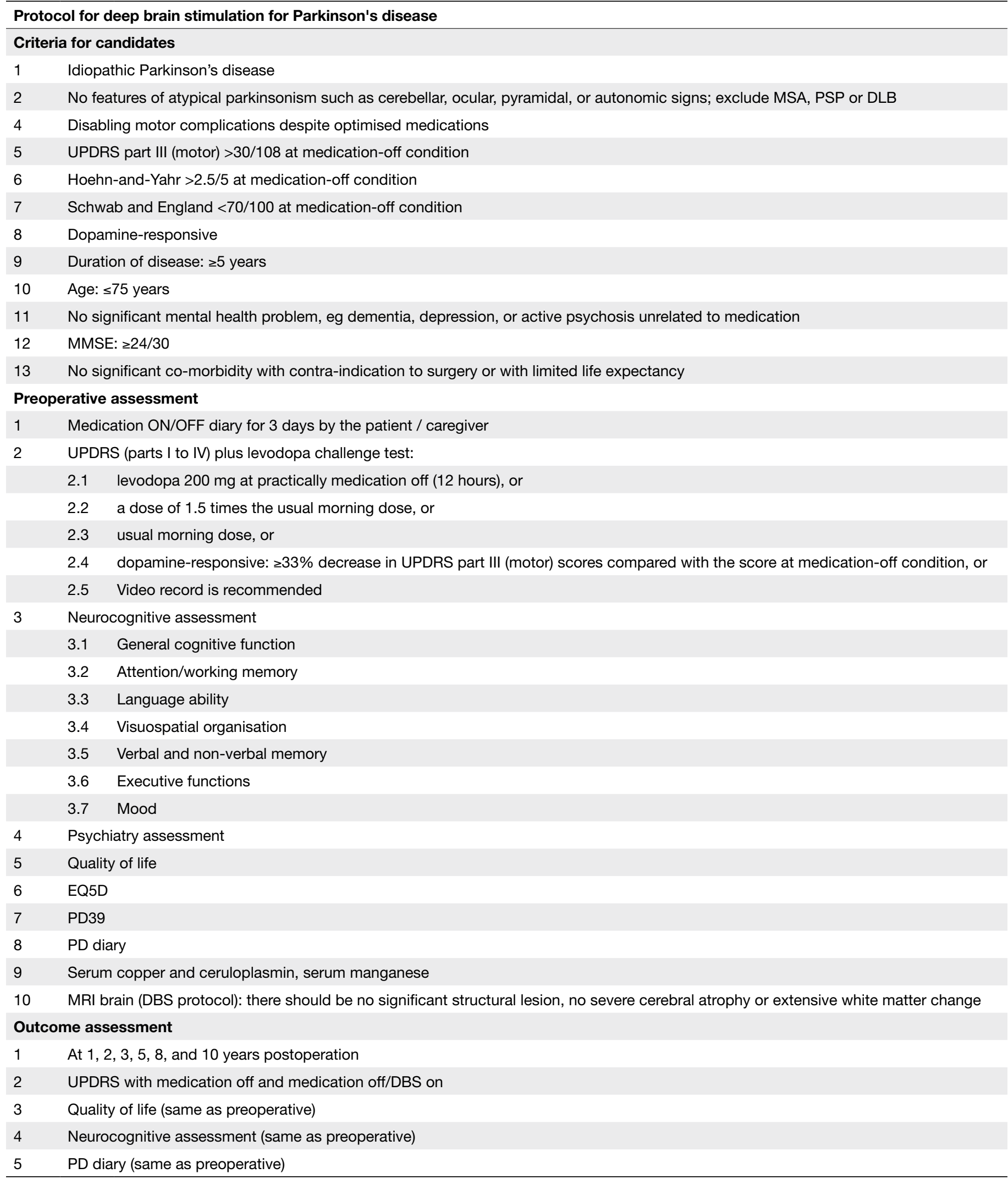

Abbreviations: DBS = deep brain stimulation; DLB = dementia with Lewy bodies; EQ5D = EuroQOL-5 dimension questionnaire; MMSE = Mini-Mental State Examination; $\mathrm{MRI}=$ magnetic resonance imaging; MSA = multiple system atrophy; PD = Parkinson's disease; PD39 = 39-Item Parkinson's disease questionnaire; PSP = progressive supranuclear palsy; UPDRS= Unified Parkinson's Disease Rating Scale 
The UPDRS part II on activities of daily living indicated significant improvement, in terms of total rating, writing, and freezing of gait. Apart from objective assessment, patients' self-evaluation from their own PD diary also revealed improvements in terms of more 'good-on' state, mobile without disabling dyskinesia, as well as less off-period compared with those after DBS.

Since our first case of DBS for PD in 1998, a multidisciplinary team comprising a neurologist, neurosurgeon, nurse specialist, radiologist, clinical psychologist, occupational therapist, physiotherapist, and speech therapist was gradually built up. From 2000 to 2004, important evolvement of our DBS protocol included: a monthly Combined Movement Disorders outpatient clinic run by a neurologist, neurosurgeon, and nurse specialist; regular case conference; dopamine challenge test; MER; acquirement of a stereotactic frame together with a computer planning system for imaging targeting and trajectory planning; and appointment of a dedicated person for DBS programming. Our experience also increased considerably in the areas of systematic auditing of targeting accuracy, surgical complications, and clinical outcome. ${ }^{5}$ All these serve to explain the significant improvements in the outcomes after 2005 as shown in the Figure. Our current DBS protocol since 2009 for PD is shown in Table 4.

We found a non-significant trend in reduction in the dose of anti-Parkinsonian medications in terms of levodopa-equivalent medications after STN DBS. This possible reduction in medication usage is a common finding in previously published reports ${ }^{3}$ and indicates increased independence from pharmacological treatment after the surgery. We also showed that weight gain was significant after STN DBS, which is consistent with findings in the current literature. There have been plenty of studies looking into the possible mechanisms, ${ }^{8}$ but the exact causes remain uncertain. Preliminary data show that weight gain might even be more commonly encountered in STN as compared with GPi DBS in PD, suggesting additional factors in STN stimulation. ${ }^{9}$

The complication rate in our study is comparable with that in another DBS centre with $8.6 \%$ hardware-related complication. ${ }^{10}$

There are limitations in our study. This was not a prospective study. Although the data were collected prospectively, the protocol itself was continuously evolved. Our patients were not assessed uniformly at the exact same time-points postoperatively; the timing of postoperative evaluation ranged from 2 months to 77 months (mean \pm SD, $17.0 \pm 15.4$ months), which is a rather wide range. This was related to the logistic arrangements for patients to have overnight admission for ensuring 12 hours off-medication and availability of beds in a busy tertiary emergency hospital. Quality of life (eg 39-item Parkinson's disease questionnaire) is an important component of outcome assessment but it was not used in this cohort. There was no systematic neuropsychology assessment until 2008. Despite all these shortcomings, our study is the first series in Hong Kong reporting the treatment outcomes as well as the experience gained over 12 years in bilateral STN DBS for PD patients.

\section{Conclusions}

Bilateral STN DBS for PD patients having motor disabilities refractory to medical treatment showed significant improvement in motor performance and functional state, except for speech, during an observation period of up to 77 months. The surgical procedure was shown to be safe, with no perioperative mortality. Patients were found to consume less anti-Parkinsonian medications and reported less dyskinesia, but had increased body weight. A dedicated multidisciplinary team building, refinement of protocol for patient assessment and selection, improvement of targeting methods, meticulous surgical technique, and experience in programming are the key factors that contributed to the improved outcomes.

\section{References}

1. Chan DT, Mok VC, Poon WS, Hung KN, Zhu XL. Surgical management of Parkinson's disease: a critical review. Hong Kong Med J 2001;7:34-9.

2. Moro E, Lozano AM, Pollak P, et al. Long-term results of a multicenter study on subthalamic and pallidal stimulation in Parkinson's disease. Mov Disord 2010;25:578-86.

3. Schüpbach WM, Chastan N, Welter ML, et al. Stimulation of the subthalamic nucleus in Parkinson's disease: a 5 year follow up. J Neurol Neurosurg Psychiatry 2005;76:1640-4.

4. Krack P, Batir A, Van Blercom N, et al. Five-year follow-up of bilateral stimulation of the subthalamic nucleus in advanced Parkinson's disease. N Engl J Med 2003;349:1925-34.

5. Chan DT, Zhu XL, Yeung JH, et al. Complications of deep brain stimulation: a collective review. Asian J Surg 2009;32:258-63.

6. Zhou JY, Yu Y, Zhu XL, Ng CP, Lu G, Poon WS. Parkinson's disease: insights from the laboratory and clinical therapeutics. In: Nagata T, editor. Senescence. InTech 2012; 587-616.

7. Narayana S, Jacks A, Robin DA, et al. A noninvasive imaging approach to understanding speech changes following deep brain stimulation in Parkinson's disease. Am J Speech Lang Pathol 2009;18:146-61.

8. Montaurier C, Morio B, Bannier S, et al. Mechanisms of body weight gain in patients with Parkinson's disease after subthalamic stimulation. Brain 2007;130:1808-18.

9. Sauleau P, Leray E, Rouaud T, et al. Comparison of weight gain and energy intake after subthalamic versus pallidal stimulation in Parkinson's disease. Mov Disord 2009;24:2149-55.

10. Baizabal Carvallo JF, Mostile G, Almaguer M, Davidson A, Simpson R, Jankovic J. Deep brain stimulation hardware complications in patients with movement disorders: risk factors and clinical correlations. Stereotact Funct Neurosurg 2012;90:300-6. 